Oper Orthop Traumatol 2015 $\cdot 27: 210-220$ DOI 10.1007/s00064-015-0402-z

Eingegangen: 6. September 2014

Überarbeitet: 5. November 2014

Angenommen: 12. November 2014

Online publiziert: 3. Juli 2015

(c) Springer-Verlag Berlin Heidelberg 2015

Redaktion

D.C. Wirtz, Bonn

Zeichner

R. Himmelhan, Mannheim

K. Ziebarth $\cdot$ T. Slongo

Abteilung für Kinder-Traumatologie und -Orthopädie, Universitäts Kinderklinik Bern, Bern, Schweiz

\title{
Osteotomien am proximalen Femur mit der winkelstabilen kindlichen Hüftplatte (LCP): Valgusosteotomie
}

der kanülierten Winkelplatte etwas erleichtert (CAPOS System, Fa. Synthes, Luzernstr. 21, 4528 Zuchwil, Schweiz). Seit 2007 ist die pädiatrische Hüftplatte (Fa. Synthes) als winkelstabiles Implantat erhältlich und hat sich als zuverlässiges Implantat für diese Operationen erwiesen.

Ein Nachteil der Fixationstechnik mit einer Klingenplatte war sowohl ein relativ großer Knochenverlust durch das Einbringen der Klinge in den Schenkelhals, als auch das relative Vorstehen der Klingenplatte mit konsekutiver Weichteilirritation. Des Weiteren konnte nach Platzierung der Klinge im Schenkelhals eine Korrektur der Plattenlage nur mehr sehr problematisch vorgenommen werden, da aufgrund des Substanzverlusts durch ein erneutes Einbringen der Klinge die stabile Fixation der Klinge im Schenkelhals nicht mehr gewährleistet war. Praktisch war dies bei bereits erfolgter Osteotomie nicht mehr möglich. Bei der Anwendung der winkelstabilen Kinderhüftplatte ist diese Problematik des Knochenschadens oder Substanzverlusts durch das Einbringen eines $2 \mathrm{~mm}$ dünnen Zieldrahts, der im Falle einer Fehlplatzierung ohne ossären Schaden neu platziert werden kann, nicht mehr gegeben. Aus diesem Grunde ist die Paediatric Hip Plate sehr gut zur Fixation des Knochens nach proximalen Femurosteotomien beim Kind geeignet.

Die folgende Beschreibung der Operationstechnik bezieht sich auf die Anwendung der Valgus LCP Pädiatrie-Hüftplatte 3.5/5.0.

\section{Operationsprinzip und -ziel}

Bei der proximalen Femurosteotomie wird das koxale Femurende inter-/ subtrochantär durchtrennt, um danach den Collum-Centrum-DiaphsenWinkel (CCD), die femorale Anteversion oder die Flexion/Extension des proximalen Femurs so einzustellen, wie es die zugrundeliegende Erkrankung/Deformität zur Optimierung des Containments erfordert. Im Falle einer Valgisation wird ein pathologisch niedriger CCD-Winkel normalisiert oder eine zusätzliche Erhöhung des CCD-Winkels erzielt, um z. B. bei Pseudarthrosen des Schenkelhalses die Kompressionskräfte zu erhöhen und die Scherkräfte für eine korrekte Ausheilung der Pseudarthrose zu minimieren. Die Operationstechnik gewährt eine stabile Osteosynthese des Knochens ohne Korrekturverlust und erlaubt eine funktionelle Nachbehandlung.

\section{Vorteile}

- Anatomisches Plattendesign erleichtert die Platzierung am proximalen Femur

- Keine/wenig Weichteilirritation

- Stabile Osteosynthese erlaubt funktionelle Nachbehandlung ohne Gips

- Winkelstabilität vermindert das Risiko des Korrekturverlusts auch bei schlechter Knochenqualität

- Operationstechnik einfach, mehrfache Korrekturen sind ohne Stabilitätsverlust intraoperativ möglich 

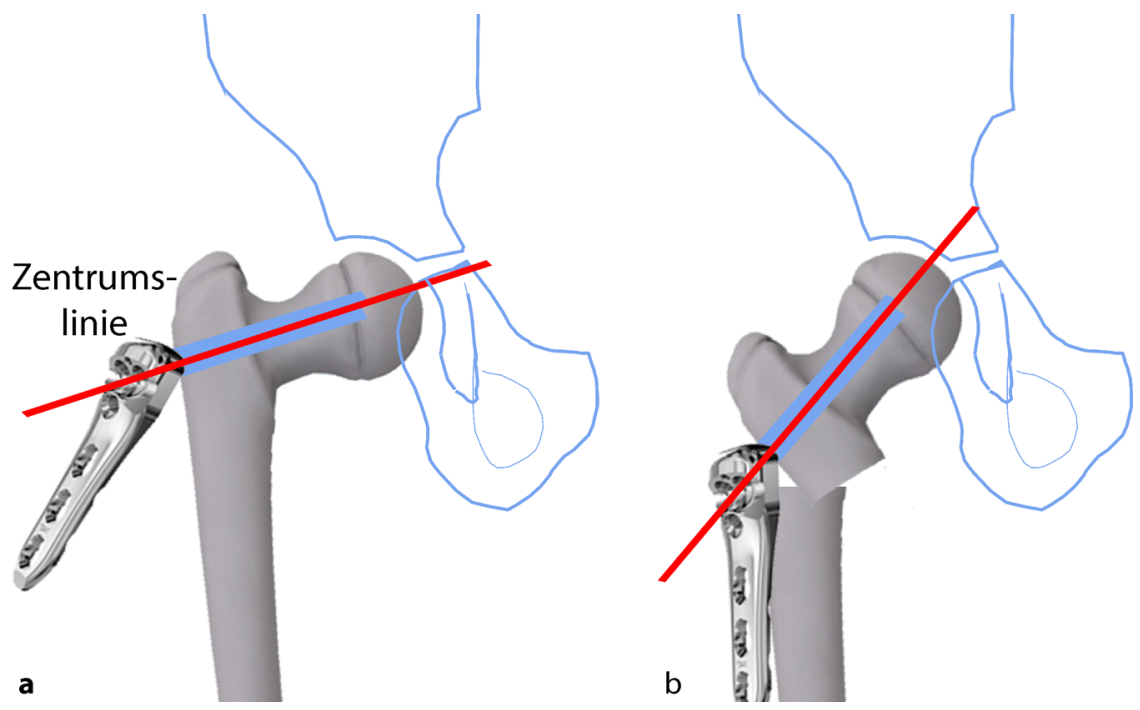

Abb. 1 A Center-Center-Technik: a Varischer CCD Winkel. b Korrektur gemäß Plattendesign ( $140^{\circ}$ Valgus). Die Schrauben werden parallel zur Achse des Schenkelhalses eingebracht
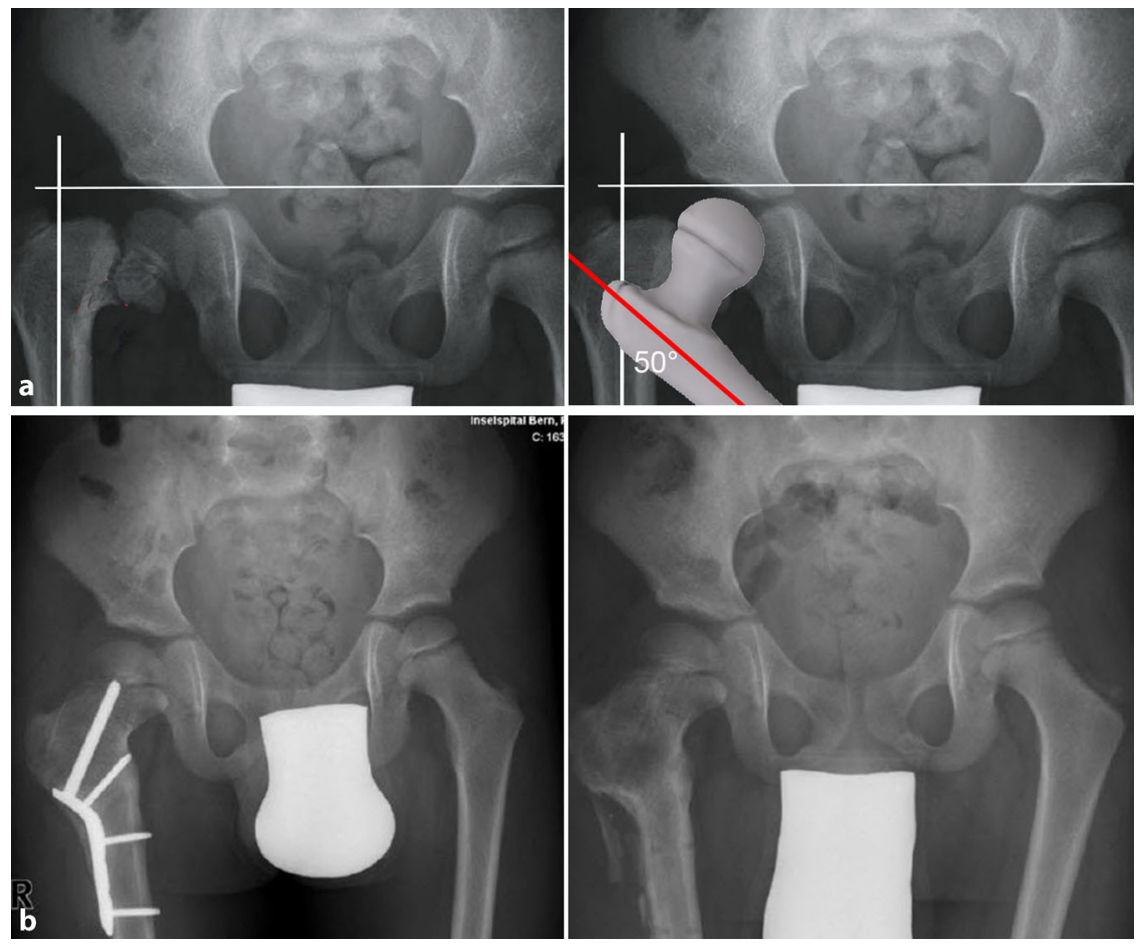

Abb. $2 \Delta$ a Präoperative Planung zur funktionellen Bestimmung des Korrekturwinkels bei Valgisation. Auf der Adduktionsaufnahme (rechtes Bild) Messung der benötigten Adduktion, bis der Hüftkopf in der gewünschten Ebene positioniert ist, wie in diesem Beispiel um die Pseudarthrose zu horizontalisieren. Hier kann das Ausmaß der Adduktion in Grad als Korrekturwinkel bestimmt werden. $\mathbf{b}$ Drei Monate postoperativ Ausheilung der Pseudarthrose und der Osteotomie (linkes Bild). Zustand nach Osteosynthesematerialentfernung 1 Jahr postoperativ (rechtes Bild)

- Zahlreiche Schraubenlängen erlauben eine hohe Variabilität für die entsprechende Schenkelhalslänge

\section{Nachteile}

- Bei adäquater und korrekter Anwendung und Umsetzung der Operationstechnik waren bis heute weder operationstechnisch noch implantatbezogene Nachteile zu erkennen

- Hohe Kosten des Implantats

\section{Indikation}

- Kongenitale Erkrankungen (z. B. residuelle Hüftdysplasie, Coxa vara congenita

- Erworbene Fehlstellungen (z. B. Pseudarthrose des Schenkelhalses nach Fraktur, Zustand nach Epiphysolysis capitis femoris, Zustand nach Morbus Perthes)

\section{Kontraindikationen}

Im Falle von leichtgradigen/schweren Hüftdysplasien mit marginaler Überdachung des Hüftkopfes vor Valgisation, sollte gleichzeitig eine Korrektur am Becken (Osteotomie/Acetabuloplastik) durchgeführt werden, um eine Subluxation des Femurkopfes zu vermeiden.

\section{Patientenaufklärung}

- Es ist überaus wichtig, den Eltern des häufig noch beschwerdefreien Kindes (z. B. bei residueller Hüftdysplasie) das Ziel der Operation (meistens eine langfristige Verbesserung der Situation und Vermeidung einer sekundären Coxarthrose) verständlich zu erklären.

- Allgemeine Operationsrisiken

- Infektion der Operationswunde, bei welcher eine Antibiotikatherapie oder manchmal sogar eine Wundrevision erforderlich wird

- Nachblutung, welche ebenfalls selten eine operative Hämatomentlastung erfordert

- Schnittführung (Narbe), gelegentlich hypertrophe Narbenbildung

- Spezielle Operationsrisiken

- Verletzung von benachbarten Nerven und Blutgefäßen (N. ischiadicus, A./V. femoralis)

- Materialbruch/-versagen

- Nachbehandlung

- Postoperative Nachbehandlung (Mobilisation mit 5-10 kg Teilbelastung etc.) 
Oper Orthop Traumatol 2015 - 27:210-220 DOI 10.1007/s00064-015-0402-z

(c) Springer-Verlag Berlin Heidelberg 2015

\section{K. Ziebarth · T. Slongo}

\section{Osteotomien am proximalen Femur mit der winkelstabilen kindlichen Hüftplatte (LCP): Valgusosteotomie}

\section{Zusammenfassung}

Operationsziel. Sichere und suffiziente proximale Femurosteotomie mit niedriger Komplikationsrate durch exakte und zuverlässige präoperative Planung. Indikationen. Congenitale Schenkelhalspseudarthrose. Posttraumatische Schenkelhalspseudarthrose. Coxa vara. Kontraindikationen. Keine. Bei bestimmten Pathologien wie z. B. der Hüftdysplasie sind alleinige Korrekturen des proximalen Femurs gerade bei älteren Kindern häufig nicht ausreichend und sollten mit einer Beckenosteotomie kombiniert werden. Operationstechnik. Exakte präoperative Planung der gewünschten Korrektur. Lateraler
Zugang zum proximalen Femur. Platzieren eines Anteversionsdrahts auf den ventralen Schenkelhals. Setzen des Positions-KirschnerDrahts. Einbringen der 2,8-mm-KirschnerDrähte in den Schenkelhals. Osteotomieren des proximalen Femurs nach Markierung der Rotation mit Kirschner-Drähten oder der oszillierenden Säge. Schieben der Platte über die Kirschner-Drähte und Fixation der Platte an das proximale Fragment durch Austauschen der Kirschner-Drähte mit winkelstabilen Schrauben. Reposition des Femurschafts an die Platte und Fixation mit winkelstabilen oder Kortikalisschrauben. Readaptation des Muskels über der Platte.
Weiterbehandlung. Je nach verwendeter Platte ist keine äußere Fixation notwendig. Mobilisation an Gehstöcken mit Teilbelastung je nach Alter für 4-6 Wochen.

Ergebnisse. In einer eigenen Publikation suffiziente Korrektur und stabile Osteosynthese bei 22 Patienten (30 Hüften). Niedrige Komplikationsraten. Die exzellenten Ergebnisse wurden auch durch weitere Studien unterstützt.

\section{Schlüsselwörter}

Coxa vara - Congenitale Schenkelhals-Pseudoarthrose · Posttraumatische SchenkelhalsPseudoarthrose

\section{Proximal Femoral Osteotomies with the Paediatric Hip Plate (LCP): Valgus osteotomy}

\section{Abstract}

Objective. Proximal femoral osteotomy with stable fixation and sufficient correction. Low complication rates due to exact preoperative planning.

Indications. Congenital or traumatic femoral neck pseudarthrosis. Coxa vara.

Contraindications. None. In severe deformities, a single femoral osteotomy may not solve the problem; thus, additional correction, e.g., a pelvic osteotomy, is required.

Surgical technique. Correct planning of the correction angle. Lateral approach. Subperiosteal detachment of vastus lateralis muscle. Place guide wire on the femoral neck to judge anteversion. Insert positioning wire $5 \mathrm{~mm}$ distal to trochanteric physis. Insert $2.8 \mathrm{~mm}$ Kirschner wire in the femoral neck. Osteotomy of the femur after marking the rotation by Kirschner wires or oscillating saw. Slide LC plate over Kirschner wires. Replace Kirschner wires with screws. Reduction of the femoral shaft to the plate with bone forceps. Definitive fixation of the plate to the femoral shaft by cortex or locking screws. Readaptation of vastus lateralis muscle over the plate.

Postoperative management. Partial weightbearing for 4-6 weeks depending on the age of the patient without any external fixation (e. g. cast) is possible.

Results. Recent studies support the authors' findings of sufficient correction and stable fixation after proximal femoral osteotomy with the LCP pediatric hip plate. Low complication rates and stable fixation.

\section{Keywords}

Coxa vara - Congenital pseudarthrosis of the femoral neck - Posttraumatic pseudarthrosis of the femoral neck
- Postoperativer Schmerz (Schmerzbehandlung mit Schmerzpumpe, Regionalanästhesie etc.)

- Dauer der postoperativen Hospitalisation (i. d. Regel 3-5 Tage)

- Materialentfernung nach vollständiger Konsolidation der Osteotomie

\section{Operationsvorbereitung}

- Präoperatives Routinelabor mit Blutbild, Leukozyten, Thrombozyten und Quickwert. Je nach Komorbidität und vor allem bei behinderten Patienten Bestimmung von Elektrolyten,
Nierenwerten und Gerinnungsstatus inklusive PTT und Thrombinzeit

- Keine Blutkonserven notwendig

- Standardisierte Nativröntgenbildgebung

- a.-p.-Röntgenbild des Beckens sowie entweder Abduktions-Innenrotationsaufnahme (Varuskorrektur) oder Adduktionsaufnahme (Valguskorrektur) nicht mehr als 6 Monate zurückliegend

- Ggf. MRI oder CT, je nach Indikation der Operation

- Sicherstellung der korrekten Lagerung des Patienten; je nach Größe des Patienten und Vorliebe des Operateurs in Rücken- oder Seitenlagerung. Bei Rückenlage Verfügbarkeit eines röntgendurchlässigen Operationstischs nötig

\section{Operationsplanung und}

Auswahl der korrekten

Größe und Angulation der

Osteosyntheseplatte

- Center-Center-Technik (• Abb. 1):

Plattenwinkel definiert die Korrektur respektive den erreichten postoperativen Collum-CentrumDiaphysenwinkel (CCD). Generell wird die Valgusosteotomie immer in 


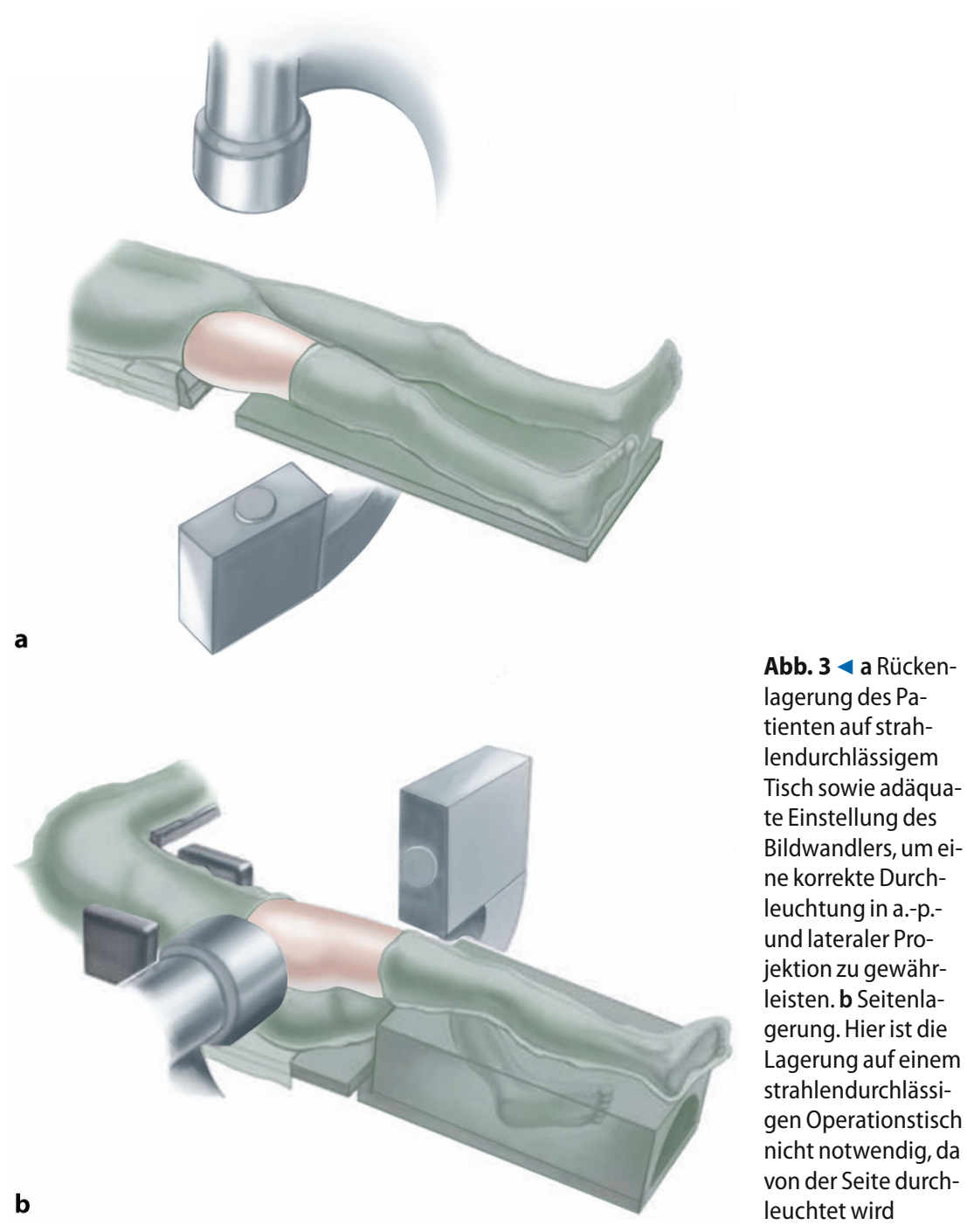

der Center-Center-Technik geplant um eine größtmögliche Valguskorrektur zu erzielen. Die funktionelle Bestimmung ( $\bullet$ Abb. 2a, b) bei der Valguskorrektur dient lediglich dazu, approximativ einzuschätzen wieviel Valguskorrektur für die Hüfte in etwa benötigt wird. Die funktionelle Planung kommt eher in der offenen hüftgelenkserhaltenden Chirurgie (Chirurgische Hüftluxation) zur Anwendung und dient dazu einzuschätzen, wieviel Korrektur es benötigt um den guten Hüftkopfanteil in die Hauptbelastungszone einzustellen.

- Funktionelle Bestimmung

(- Abb. 2a, b)
- Valguskorrektur: Notwendige Adduktion in der Adduktionsaufnahme oder der Arthrographie, in der die gewünschte Korrektur erreicht wird: Der Kopf-Schenkelhals-Winkel ist wieder auf einem physiologischen Wert zwischen 120-130 eingestellt oder der Frakturspalt verläuft $z$. B. im Falle einer Pseudarthrose perpendikulär zur Belastungsachse, um eine optimale Kompression der Fraktur oder Pseudarthrose zu erzielen und Scherkräfte zu minimieren. Die endgültige Berechnung des Korrekturwinkels wird nicht selten erst im Operationssaal durchgeführt, indem bei dem bereits anästhesierten Patienten mittels Arthrographie oder nach Arthrotomie bei offenem Verfahren, die optimale Einstellung des Hüftkopfs gesucht wird.

- Valgisation (Beispiel): Präoperativer CCD $90^{\circ}$, optimale Einstellung des Hüftkopfs bei $50^{\circ}$-Adduktion, d. h. Korrekturwinkel $50^{\circ}$. Verwendung der $140^{\circ}$-Valgusplatte. Einstellung des Zielgeräts somit $140^{\circ}-50^{\circ}=90^{\circ}$. Nach Frakturen oder bei Coxa vara congenita wird häufig eine Valgisation auf 140-150 ${ }^{\circ}$ angestrebt (• Abb. 2a, b).

\section{Implantate und Instrumentarium}

- Ein standardisiertes Hüftsieb mit Langenbeck und Hohmann Hebeln

- Raspatorium

- Lexermeissel 10/23 mm

- Oszillierende Säge

- Standardinstrumente für die pädiatrische Hüftplatte [1]

- Verfügbare Plattenmodelle:

- LCP 3,5 mm (Verwendung bis 35 kg Körpergewicht): $140^{\circ}$-Valgusplatte

- LCP 5,0 mm (> 35 kg Körpergewicht): $140^{\circ}$-Valgusplatte

\section{Anästhesie und Lagerung}

- Intubationsnarkose

- Maximale Relaxation des Patienten erleichtert die Manipulation der Fragmente

- Je nach Größe und Gewicht des Patienten sowie Vorliebe des Operateurs Rücken- oder Seitenlagerung möglich (• Abb. 3a, b)

- Gute Fixation des Patienten mit Seitenstützen etc.

- Im Falle von Rückenlage: Anheben des Beckens durch Unterlage von Tüchern unter das Os sacrum erleichtert die Präparation sowie das Einbringen der Zieldrähte

- Korrekte Platzierung des Bildwandlers zur Visualisierung des Operationsgebiets in 2 Ebenen (• Abb. 3a, b) 


\section{Operative Techniken}

\section{Operationstechnik}

(• Abb. 4, 5, 6, 7, 8, 9, 10, 11, 12, 13, 14, 15)

Auf die Varisation wurde bereits detailliert in einer früheren Ausgabe von Operative Orthopädie und Traumatologie [1] eingegangen.

Der hier beispielhaft gezeigte Fall betrifft einen 6-jährigen Jungen mit Coxa vara bei Schenkelhalspseudarthrose (Ge- wicht $30 \mathrm{~kg}$, Varusstellung der Hüfte $90^{\circ}$, Abb. 2a). Operation: Valgisation von $50^{\circ}$ auf einen Winkel von $140^{\circ}$ zur Horizontalisierung der Pseudarthrose und Kompression des Pseudarthrosespalts (- Abb. 2b). Entsprechend dem Gewicht des Patienten und des Korrekturwinkels wird die 3,5-mm-140 Valgusplatte ausgewählt. Das Zielgerät muss gemäß der präoperativen Planung auf $90^{\circ}$ eingestellt werden (Platte $140^{\circ}$ minus Korrekturwinkel $50^{\circ}=90^{\circ}$ ).

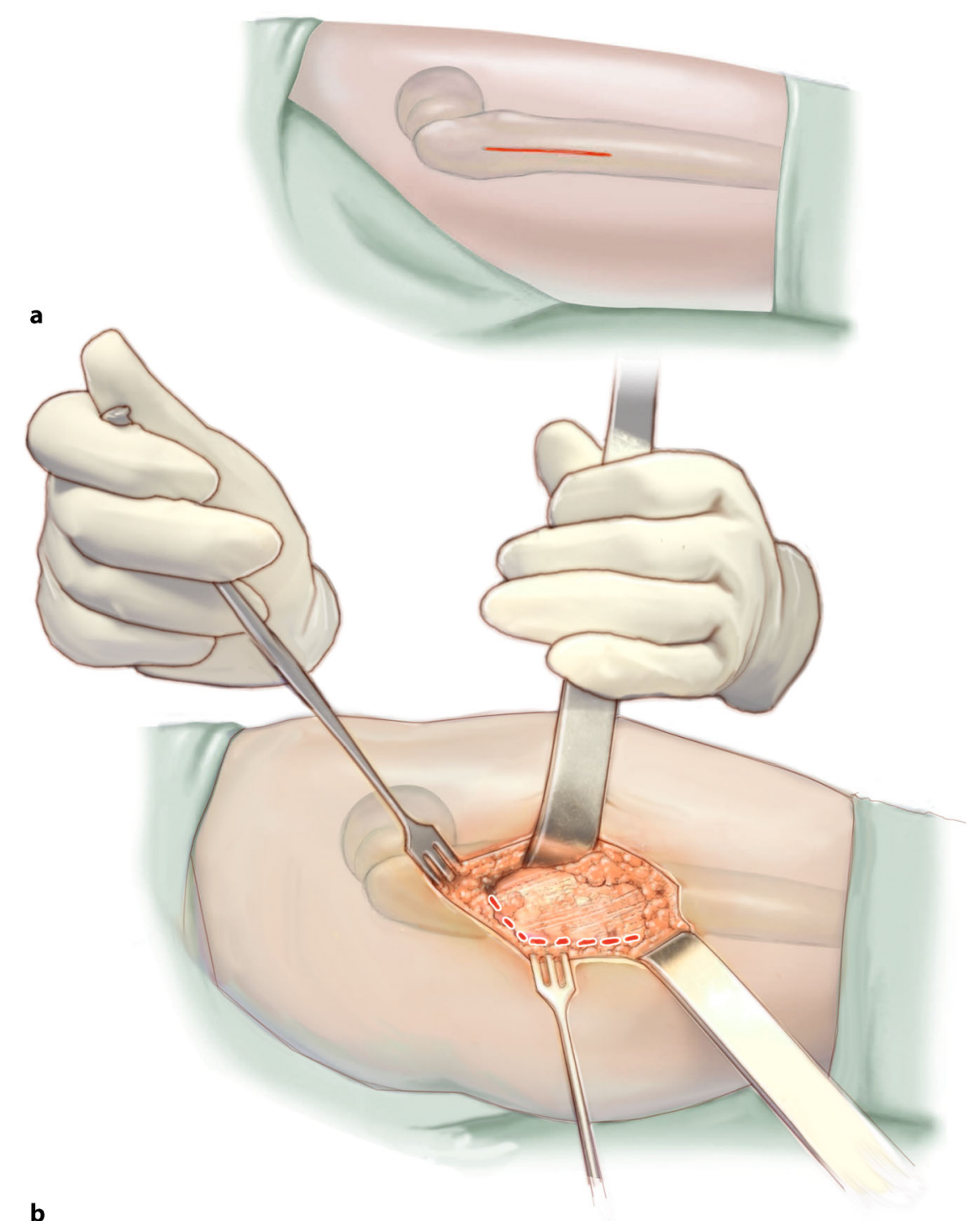

b

Abb. $4 \Delta$ a, b Zugang zum lateralen Femur durch laterale Hautinzision unterhalb des Trochanter major. Etwa $8 \mathrm{~cm}$ lange Spaltung der Tensorfaszie und schonendes Eingehen auf das Femur am Hinterrand des Vastus lateralis 


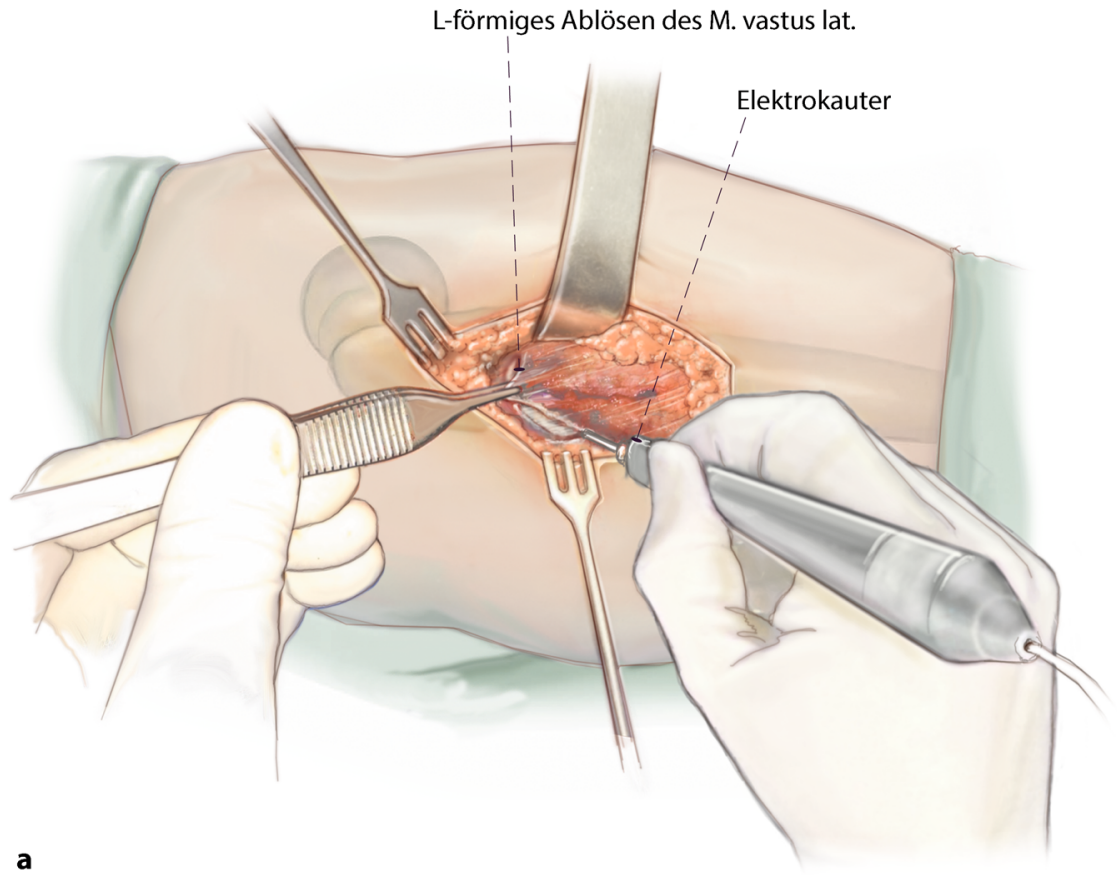

a

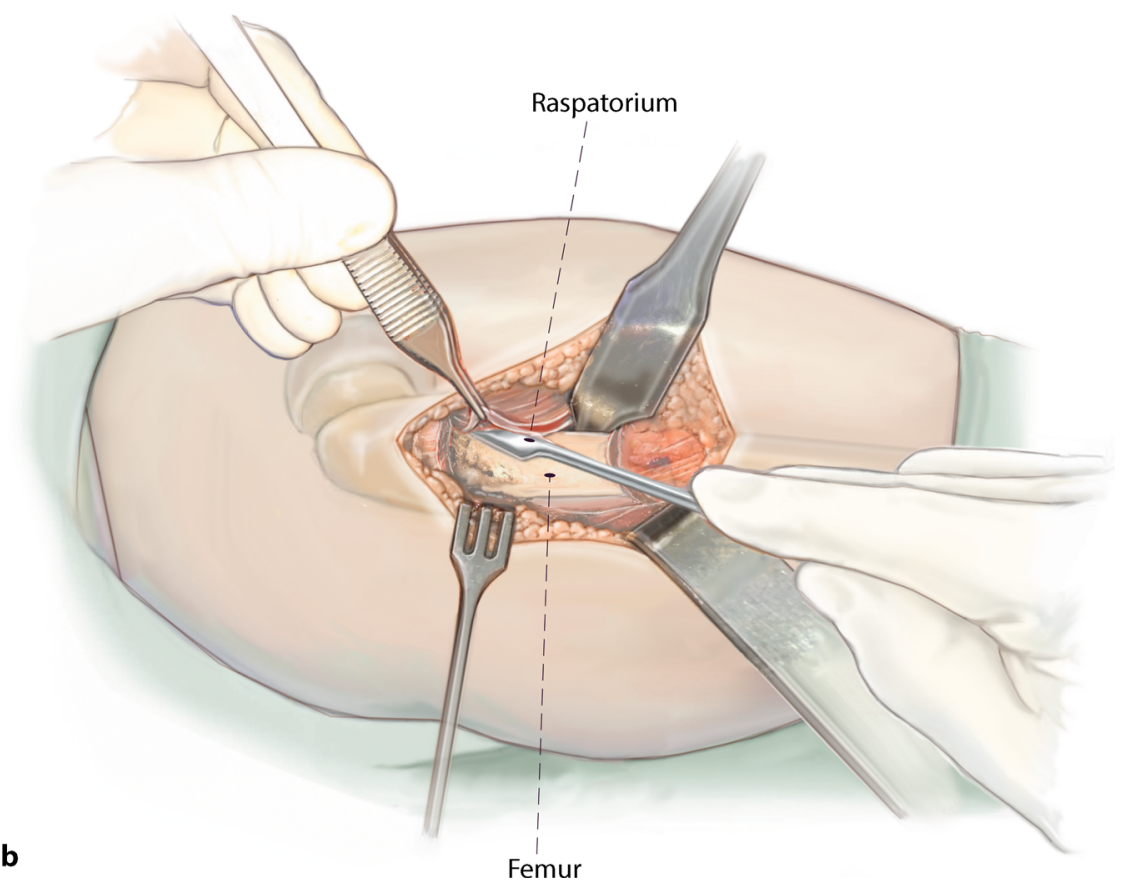

Abb. $5 \Delta$ a, b L-förmiges Ablösen des Vastus lateralis Muskels durch direkten Schnitt transperiostal direkt auf das Femur; Abheben des Periost-Muskelschlauchs mit dem Raspatorium. Darstellen des proximalen Femurs mit zwei Hohmann-Haken 


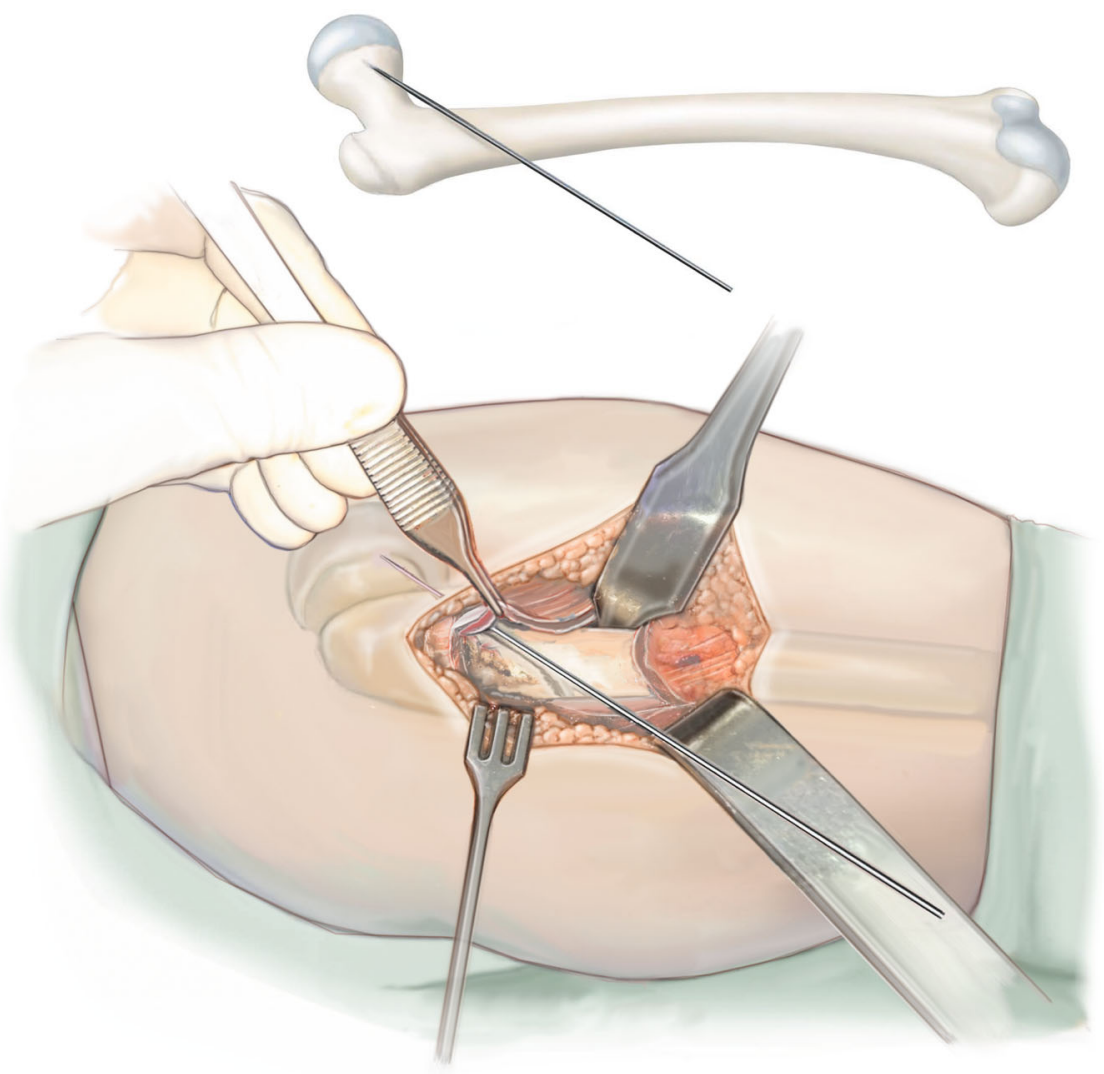

Abb. $6 \Delta$ Ein Zur Visualisierung der femoralen Anteversion wird ein 2,0-mm-Kirschner-Draht auf den ventralen Schenkelhals gelegt
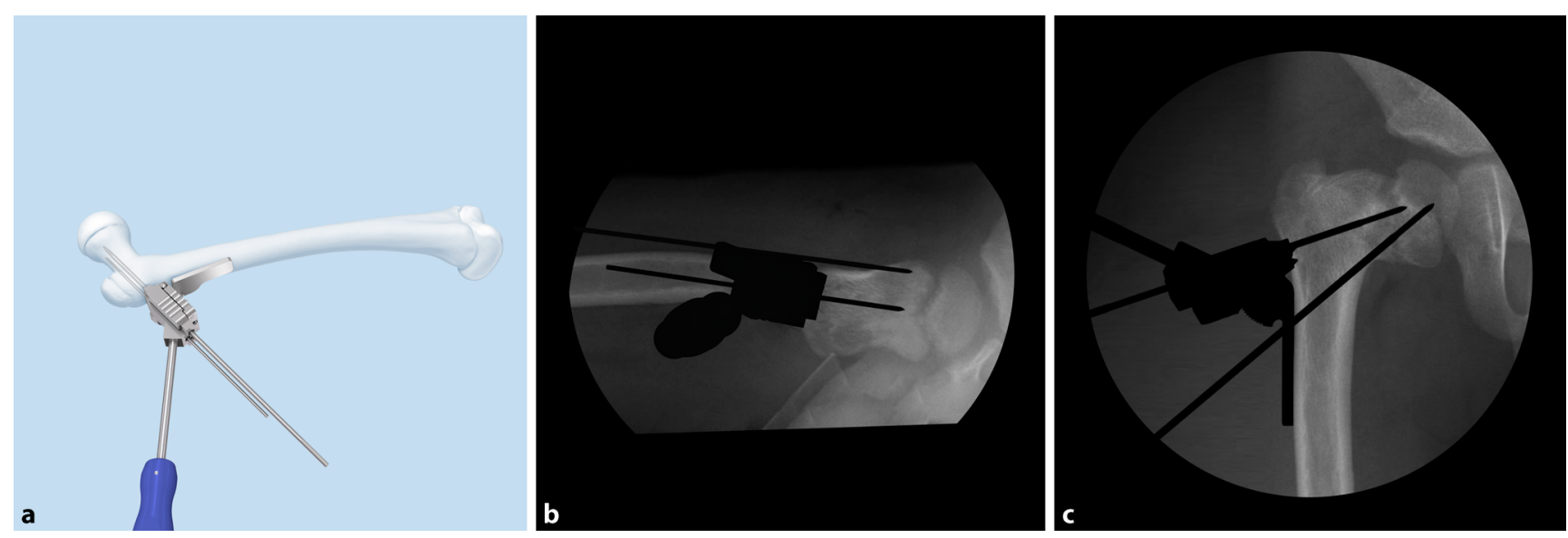

Abb. $7 \Delta$ a Der Positions-Kirschner-Draht wird 5-6 mm distal der lateralen Apophysenfuge des Trochanter major parallel zum Antetorsionsdraht in den Schenkelhals eingebracht. (Mit freundl. Genehmigung der Fa. Synthes). b, c Bildwandlerkontrolle der korrekten Lage in beiden Ebenen (a.-p. und axial). Entspricht die Stellung des Positions-Kirschner-Drahts nicht der Planung und ist er im axialen Strahlengang nicht perfekt im Zentrum, muss er neu und korrekt platziert werden. Wie oben erwähnt, ist dies ohne größeren Aufwand oder Schädigung des Schenkelhalses mehrere Male möglich. Sitzt der Positions-Kirschner-Draht korrekt, kann der Anteversionsdraht entfernt werden 


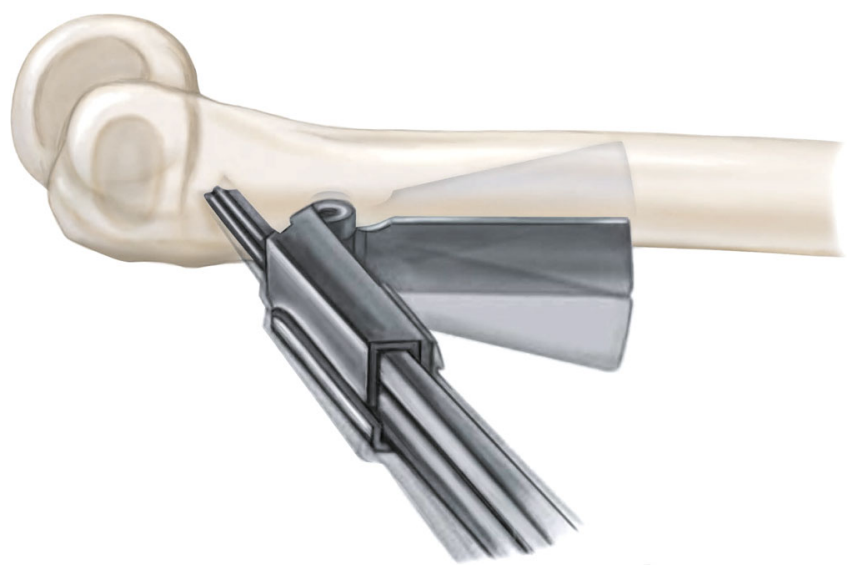

Abb. 8 A Einbringen der 2,8-mm-Kirschner-Drähte über das Zielgerät in den Schenkelhals bis ca. $5 \mathrm{~mm}$ proximal der Wachstumsfuge. Der Flügel des Zielblocks sollte beim Einbringen der Kirschner-Drähte für die LCP-Schrauben dem Femurschaft parallel und zentral anliegen. Ist gleichzeitig eine Korrektur hinsichtlich Flexion oder Extension geplant, muss der Flügel des Zielblocks entsprechend nach ventral (Flexionskorrektur) oder nach dorsal (Extensionskorrektur) geschwenkt werden

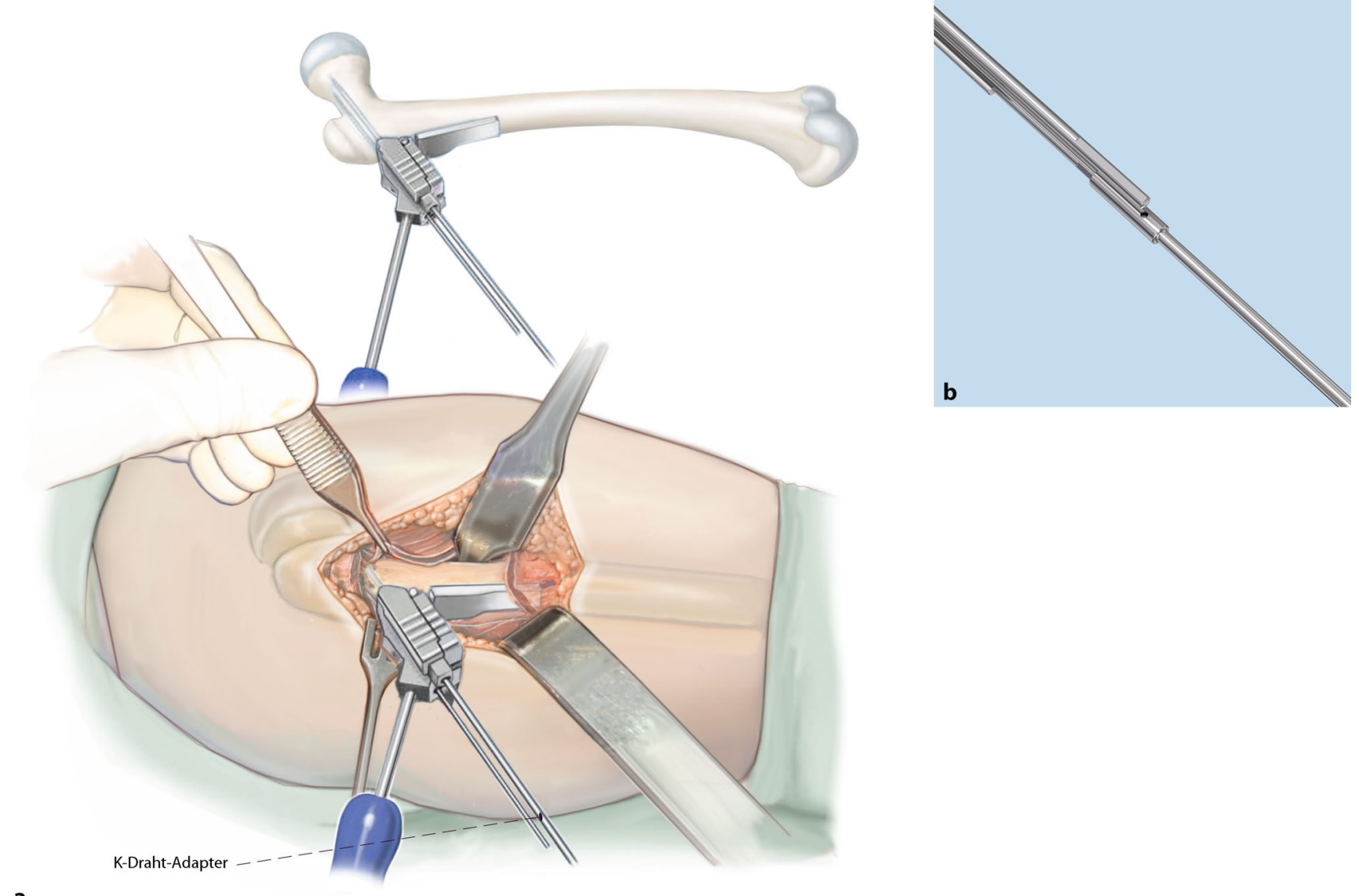

a

Abb. $9 \Delta$ a, bDerzweite Kirschner-Draht sollte mit dem Kirschner-Draht-Adapter eingebracht werden, um nicht mit der Bohrmaschine am ersten Kirschner-Draht abgelenktzu werden. (Abb.9b mit freundl. Genehmigung der Fa. Synthes). Man beachte: Auch nach dem Einbringen der beiden 2,8-mm-Schrauben-Kirschner-Drähte darf der Positionierungs-Ziel-Kirschner-Draht $(2,0 \mathrm{~mm})$ auf keinen Fall entfernt werden. Es besteht sonst die Gefahr, dass sich die Platte beim Setzen der Schrauben um den verbliebenen Kirschner-Draht dreht (siehe $-A b b .14)$. Entfernen des Zielinstrumentariums. Es empfiehlt sich aus technischen Gründen, vor allem bei sehr kurzer Inzision, die Schraube am Zielblock zu lösen, um die Entfernung desselbigen zu vereinfachen 


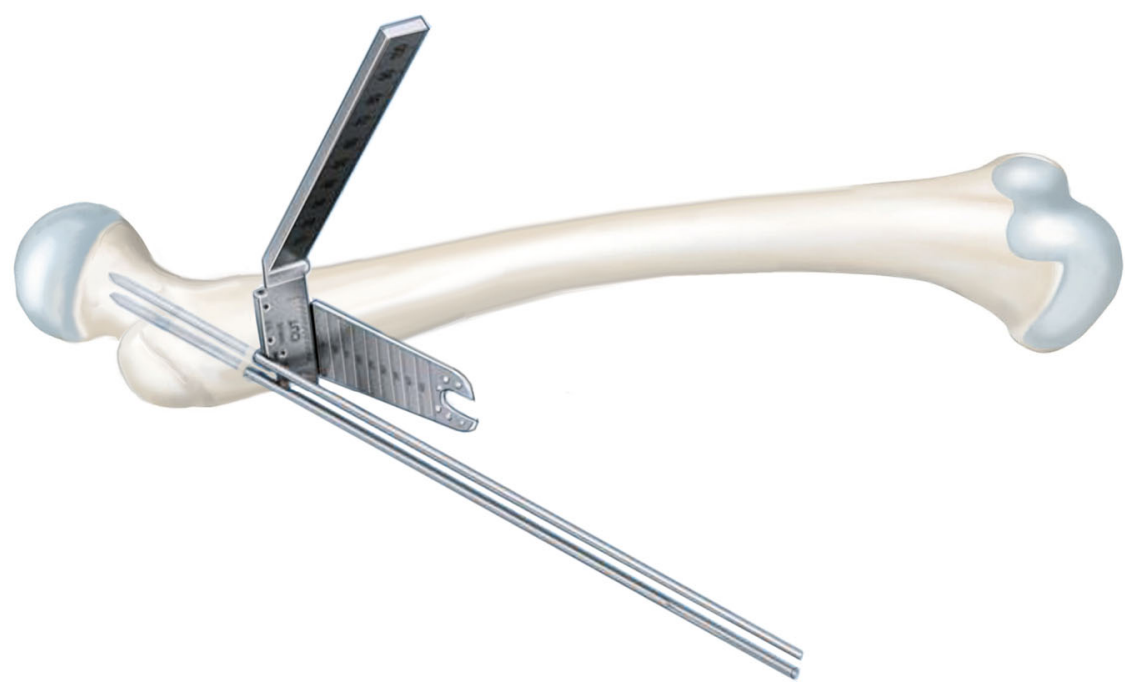

Abb. $10 \triangle$ N Nun wird mit dem Osteotomiemessgerät der ideale Abstand der Osteotomie von den LCP-Schrauben gewählt. Vom Hersteller wird als optimaler Abstand bei der 3,5-mm-LCP $18 \mathrm{~mm}$ und bei der 5,0-mm-LCP $23 \mathrm{~mm}$ empfohlen. Bei extremen Valgusfehlstellungen empfiehlt es sich, die Osteotomie etwas tiefer (3-4 mm) zu wählen, um nach der Korrektur noch Platz für die Kalkarschraube zu haben

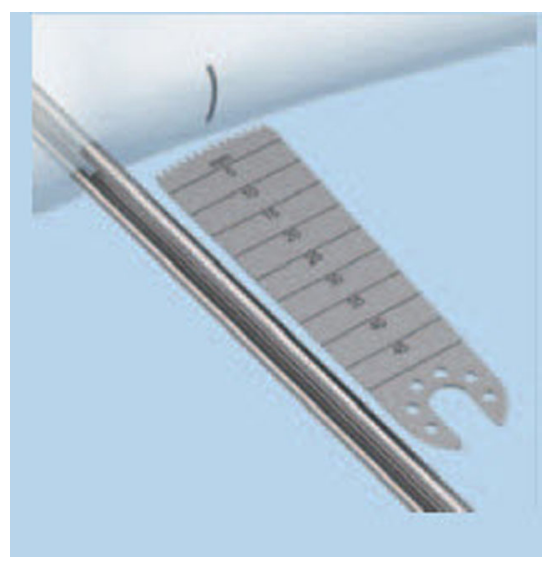

Abb. $12 \Delta$ Bei reiner Valguskorrektur kann auch optional eine oberflächliche Markierung mit der oszillierenden Säge $90^{\circ}$ zur Osteotomie angelegt werden. Vervollständigen der Osteotomie. (Mit freundl. Genehmigung der Fa. Synthes)

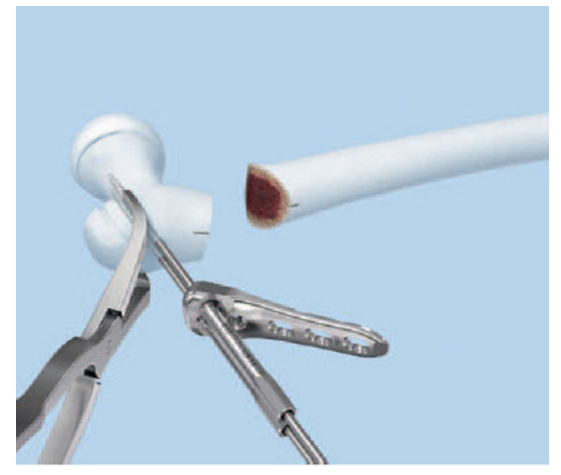

Abb. $13 \Delta$ Halten des proximalen Fragments mit der "Reduction Forceps, toothed, soft lock", der sog. Spanierzange und Einschieben der Platte über die Kirschner-Drähte. Zu beachten ist, dass vorher die Bohrhülsen auf die beiden proximalen Plattenlöcher aufgeschraubt werden müssen. Dies sollte absolut parallel und genau geschehen, da die Schrauben ansonsten nicht parallel in den Schenkelhals eingebracht werden können und eine korrekte Verblockung der Schraubenköpfe in der Platte nicht gewährleistet ist. Um einen guten und festen Sitz des feinen Gewindes zu gewährleisten, empfiehlt es sich, vordem Aufdrehen der Bohrhülsen auf die Platte sowohl die Bohrhülse selber, als auch das Gewinde in der Platte von Blut- oder Geweberesten zu reinigen. Bei der Benutzung der 5,0-mm-Platte müssen in die Bohrhülsen noch die Zentrierhülsen eingebracht werden, um keinen Korrekturverlust zu erleiden. Nach vollständigem Vorschieben der Platte messen der gewünschten Schraubenlänge mit dem direkten Längenmesser. (Mit freundl. Genehmigung der Fa. Synthes)

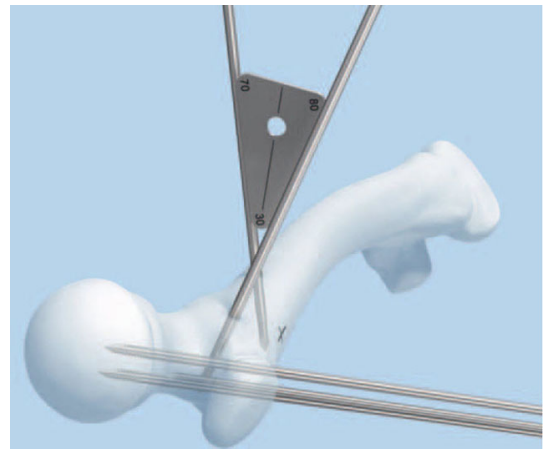

Abb. $11 \Delta$ Vor der Vervollständigung der Osteotomie sollte die Rotation mit zwei 2,0-m$\mathrm{m}$-Kirschner-Drähten proximal und distal der Osteotomie markiert werden. (Mit freundl. Genehmigung der Fa. Synthes)

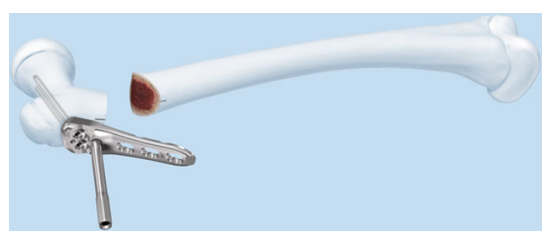

Abb. $14 \Delta$ Jeweils Entfernung eines KirschnerDrahts und Einbringen der Schraube in der gewünschten Länge ohneerneutes Bohren beiVerwendung der 3,5-mm-Platte. Nach Entfernung des Kirschner-Drahts muss bei Verwendung der 5,0-mm-Platte mit einem 4,3-mm-Bohrer das Schraubenloch in der gewünschten Länge vorgebohrt werden. Bevor nicht die beiden Schenkelhalsschrauben im Knochen platziert sind, darf der Zielkirschnerdraht aus dem Schenkelhals nicht entfernt werden, da die Platte ansonsten um die Schraube rotieren könnte. Hierdurch kann es unter Umständen zu einer Fehlplatzierung der Platte am Femurschaft mit konsekutiver Extensions- oder Flexionsfehlstellung kommen. Die Kalkarschraube wird als letzte gebohrt und nach Längenmessung winkelstabil fixiert. (Mit freundl. Genehmigung der Fa. Synthes) 


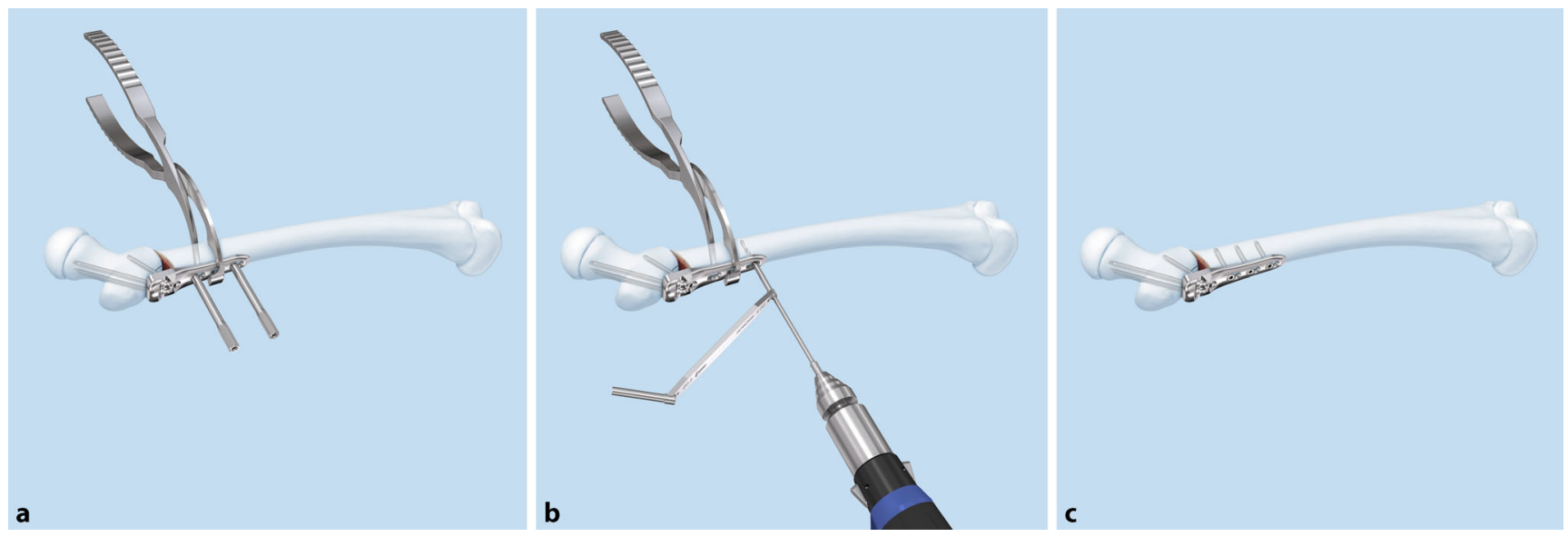

Abb. $15 \Delta$ a Reposition des Femurschafts an die Platte, am einfachsten durch Herunterdrücken der aufstehenden Platte mit dem Daumen. Danach vorläufige Fixierung der Platte am Femur mit der Verbrügge-Zange, wobei die korrekte oder korrigierte Rotation ebenfalls berücksichtigt werden muss. Sollte die Platte etwas vom Femurschaft abstehen, kann optional das mittlere Loch der Platte mit einer monokortikalen Kortikalisschraube besetzt und so die Platte an den Schaft herangezogen werden. Dieses muss natürlich unter sorgfältiger Kontrolle der Spannungsverhältnisse geschehen, um die monokortikale Schraube bei zu großem Wiederstand nicht aus dem Knochen zu brechen. Die Fixation der Platte am Schaft kann sowohl mit Kortikalisschrauben (b), als auch mit winkelstabilen Schrauben geschehen (c), wobei diese nicht miteinander kombiniert werden sollten. Der Vorteil der Verwendung von Kortikalisschrauben ist die mögliche Kompression der Osteotomie durch exzentrisches Bohren. Jedoch kann auch unter Zuhilfenahme einer Kortikalisschraube, die dann wieder entfernt wird, oder mit dem Plattenspanner eine Kompression bei derVerwendung von winkelstabilen Schrauben erzieltwerden. Bei schlechter Knochenqualität empfiehlt es sich, die winkelstabilen Schrauben am Femurschaft zu verwenden. (Mit freundl. Genehmigung der Fa. Synthes)

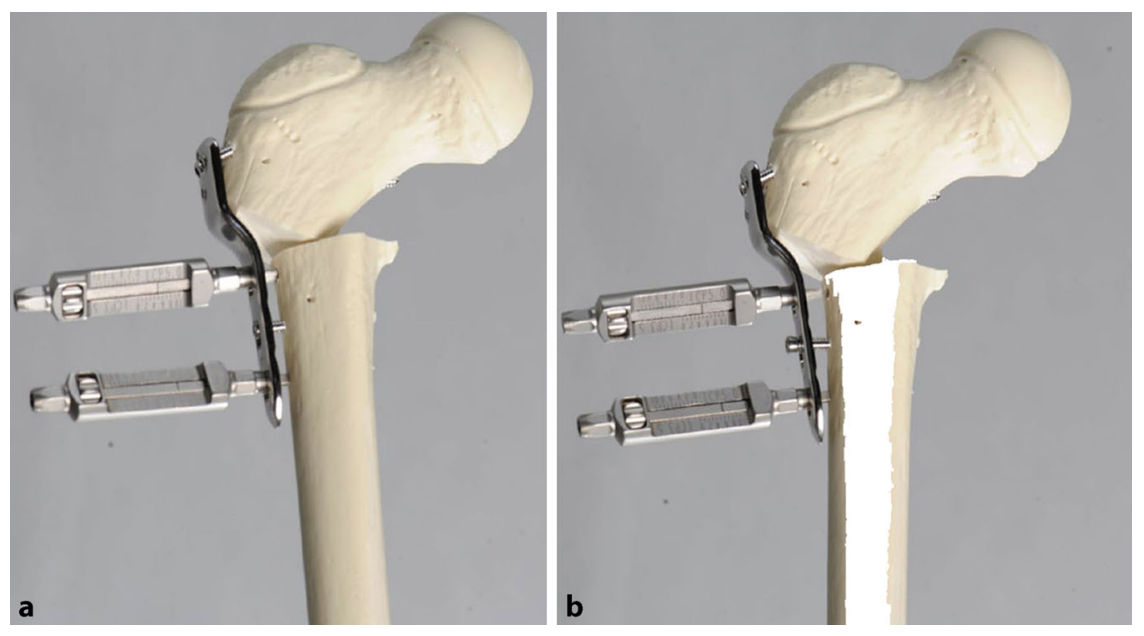

Abb. $16 \Delta$ a Je nach Ausmaß der Varus- oder Valguskorrektur kann der Femurschaft noch zusätzlich medialisiert werden. Für diesen Zweck steht ein spezielles Medialisierungsinstrument im Set zur Verfügung. b Selbst bei einer am Femurschaft bereits fixierten Platte kann im Falle einer suboptimalen Korrektur mit diesem Instrumentarium der Varus oder Valgus des proximalen Fragments um $10^{\circ}$ durch Veränderung des proximalen oder distalen Abstands der Platte am Femurschaft korrigiert werden (Hier zusätzliche Valgisation von $10^{\circ}$ ). Dies ist jedoch nur möglich, wenn am Femurschaft winkelstabile Schrauben verwendet werden

\section{Besonderheiten}

Das Plattendesign der LCP beinhaltet sowohl die Medialisierung bei Varisierungen als auch eine gewisse Lateralisation bei Valguskorrekturen (- Abb. 16a, b).

\section{Postoperative Behandlung}

- Mobilisation an Gehstöcken mit 5-10 kg Teilbelastung bei der Verwendung der 3,5-mm- und 5,0-mmPlatte (je nach Alter und Gewicht des Patienten)

- Aktive/Passive Hüftbeweglichkeit frei
- Nach 4-6 Wochen (je nach Alter des Patienten) Konsolidationsröntgenaufnahme und Übergang zur Vollbelastung

\section{Fehler, Gefahren, Komplikationen}

- Falsches Berechnen des Korrekturwinkels: Malpositionierung des Hüftkopfes (zu wenig oder zu viel Korrektur). Revisionsosteotomie.

- Falsches Drehen zur Korrektur der Rotation: Revisionsoperation. Lösen der Schrauben am Femurschaft und Veränderung der Rotation des Femurschaftes gegenüber der Platte.

- Schraubenperforation in das Gelenk: Revisionsoperation. Verwendung von kürzeren Schrauben.

- Insuffiziente Schraubenfixation (Schrauben greifen nicht vollständig im Gewinde der Platte durch inkorrektes Aufschrauben der Bohrhülsen auf die Platte): Gefahr der Schrauben/Plattenlockerung mit Korrekturverlust. Revisionsoperation mit Neuplatzierung der Schrauben.

- Verwendung von Kortikalisschrauben im Schenkelhals: Keine suffiziente Stabilität. Verlängerte Teilbelastung oder Austausch der Schrauben. 
- Infekt: Je nach Ausmass entweder Ausspülen der Wunde bis hin zur Materialentfernung und mehrfachem Debridement mit passagerer Ruhigstellung im äusseren Fixateur.

\section{Ergebnisse}

Die proximalen Femurosteotomien mit der kindlichen Hüftplatte (LCP) zeigten in einer eigenen Studie bzgl. Varusosteotomien hervorragende Ergebnisse [3]. Eine Konsolidation wurde bei 20 von 22 Patienten innerhalb von 6 Wochen beobachtet. Bei einem Patienten war eine Teilbelastung von zusätzlich 2 Monaten notwendig.

In 4 Fällen zeigten sich Schraubenlockerungen (3-mal Schaft, 1-mal Schenkelhals) ohne Relevanz.

Eine Metallentfernung wurde durchschnittlich 6 Monate postoperativ durchgeführt.

Diese guten Ergebnisse mit suffizienten Korrekturen und niedrigen Komplikationsraten konnten auch in weiteren Studien gezeigt werden $[4,5]$.

Seit der veröffentlichten Studie wurden in unserer Klinik bei ca. 50 weiteren Patienten die LCP-Platten zur Varus-/ Valguskorrektur mit identischen, sehr guten Ergebnissen angewendet.

\section{Korrespondenzadresse}

\section{K. Ziebarth}

Abteilung für Kinder-Traumatologie und -Orthopädie, Universitäts Kinderklinik Bern 3010 Bern, Schweiz

kai.ziebarth@insel.ch

\section{Einhaltung ethischer Richtlinien}

Interessenkonflikt. K. Ziebarth und T. Slongo geben an, dass kein Interessenkonflikt besteht.

Dieser Beitrag beinhaltet keine Studien an Menschen oder Tieren.

\section{Literatur}

1. Slongo TF Slongo (2008) Osteotomien am proximalen Femur bei Kindern. Oper Orthop Traumatol 20:334-353

2. Müller M (1979) Planung einer komplexen intertrochantären Osteotomie, ZOrthop 117:145-150

3. Joeris A, Audigé L, Ziebarth K, Slongo T. The Locking Compression Hip Plate: technical guide and critical analysis. Int Orthop. 2012 Nov; 36(11):2299-2306. doi: 10.1007/s00264-012-1643-1.Epub 2012 Aug 26.

4. Islam SU, Henry A, Khan T, Davis N, Zenios M (2013) The outcome of LCP Paediatric hip plate use in children with or without neuromuscular disease. Musculoskelet Surg 98(3):233-239

5. Ročák K, Poul J, Urbášek K. Accuracy of proximal femur correction achieved with the LCP paediatric hip plates. Acta Chir Orthop Traumatol Cech. 2013; 80(4):273-277. Czech.

\section{Läsionen der langen Bizepssehne, SLAP- und Bizeps-Pulley-Läsionen}

Die Funktion der langen Bizepssehne wurde vielfach klinisch, biomechanisch und elktromyographisch untersucht. Schmerzen treten vor allem durch Instabilitäten auf, die durch Läsionen verursacht werden.

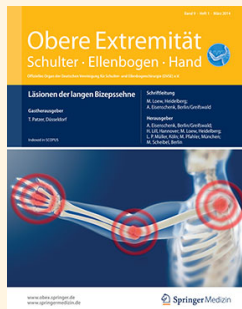
Je nach Lokalisation der Läsion werden SLAP- und Pulley-Läsionen unterschieden. Die Zeitschrift Obere Extremität geht in der Ausgabe 1/14 auf die Anatomie, Ätiologie und Diagnostik der Läsionen der langen Bizepssehne ein und stellt Therapieoptionen vor.

Lesen Sie im Leitthemenheft mehr zu folgenden Themen:

- Anatomie und Ätiologie von SLAP- und Bizeps-Pulley-Läsionen

- Diagnostik und Therapie der isolierten proximalen Bizepsläsion

- LBS-Tenotomie oder LBS-Tenodese?

- Die suprapektorale arthroskopische Tenodese

- Komplexe labrale Avulsionsverletzungen mit Extension in den SLAP-Komplex bei anterioinferiorer Schulterinstabilität

Bestellen Sie diese Ausgabe zum Preis von 52,- EUR zzgl. Versandkosten bei Springer Customer Service Center Kundenservice Zeitschriften Haberstr. 7

69126 Heidelberg

Tel.: +49 6221-345-4303

Fax: +49 6221-345-4229

E-Mail: leserservice@springer.com

Suchen Sie noch mehr zum Thema? Mit e.Med, dem Online-Paket von Springer Medizin, können Sie schnell und komfortabel in über 600 medizinischen Fachzeitschriften recherchieren.

Weitere Infos unter springermedizin.de/eMed. 